\title{
Uso da Estratégia Aprendizagem Baseada em Equipes em Disciplinas da Graduação em Farmácia
}

\author{
Emanuela Machado Silva Saraiva ${ }^{1}$, Gustavo de Oliveira Alencar ${ }^{2}$, Yanaina Samya Oliveira Machado ${ }^{3}$, \\ Antônia Edna Belém Gomes ${ }^{4}$
}

\begin{abstract}
Resumo: O objetivo da presente pesquisa foi avaliar o aproveitamento de discentes mediante a utilização da estratégia de TBL em disciplina do primeiro e do último ano da graduação em Farmácia em uma Instituição de Ensino Superior da região do Cariri cearense. Trata-se de um estudo do tipo retrospectivo, baseado na análise documental dos registros das fichas de avaliação através da estratégia de TBL aplicada em disciplinas ministradas no primeiro ano e no último ano de graduação em Farmácia. Na disciplina do primeiro ano verificou-se um acréscimo de 1,11 pontos do aproveitamento da avaliação individual do aluno para a avaliação em equipe. Já na disciplina do último ano o aumento foi de apenas 1,02 pontos. O docente deve ser capaz de inovar as abordagens em sala de aula para que o discente se sinta constantemente motivado e disposto a encarar novos desafios, como também preparar-se para trabalhar em equipe e contribuir efetivamente para o sucesso da mesma.
\end{abstract}

Palavras-chave: Aprendizagem Baseada em Equipes, Aprendizagem Ativa, Educação Farmacêutica, Farmácia.

\section{Use of Team-Based Learning Strategy in Pharmacy Graduate Disciplines}

\begin{abstract}
The aim of the present study was to evaluate students' achievement through the use of the TBL strategy in the first and last year of Pharmacy graduation in a Higher Education Institution in the Cariri region of Ceará. This is a retrospective study, based on the documentary analysis of the records of the assessment sheets through the TBL strategy applied in disciplines taught in the first year and the last year. In the discipline of the first year, there was an increase of 1.11 points of the use of the individual evaluation of the student for the evaluation in team. In the discipline of the last year of graduation in Pharmacy, there was an increase of only 1.02 points. The teacher should be able to innovate the approaches in the classroom, so that the student feels constantly motivated and willing to face new challenges, as well as preparing to work in a team and contribute effectively to the success of the same.
\end{abstract}

Keywords: Team Based Learning, Active Learning, Pharmaceutical Education, Pharmacy.

\footnotetext{
${ }^{1}$ Bacharel em FARMÁCIA pela Universidade de Fortaleza - UNIFOR (2007), com especialização em Gestão de Varejo pela Faculdades Nordeste - FANOR (2011). Em fase de conclusão do curso de pós-graduação em Docência do Ensino Superior, pela Faculdade de Juazeiro do Norte-FJN. Mestranda em Ciências da Saúde pela Faculdade de Medicina do ABC - FMABC - desenvolve pesquisa com foco em cuidados farmacêuticos na atenção básica de saúde. Com experiência em farmácia comercial, gestão em saúde e docência em saúde. Atualmente seu exercício profissional engloba as áreas de assistência e atenção farmacêutica, gestão em serviço público de saúde e docência no curso de graduação em Farmácia na cidade de Juazeiro do Norte-CE. emanuelams @ hotmail.com;

${ }^{2}$ Curso de Farmácia. Faculdade de Juazeiro do Norte - FJN. Ceará. Brasil. . gustavo_da_vinci@ hotmail.com;

${ }^{3}$ Faculdade de Juazeiro do Norte. ysamya@hotmail.com;

${ }^{4}$ Graduada em Pedagogia pela Universidade Regional do Cariri (URCA), habilitada em Língua Portuguesa pela Universidade Vale do Acaraú (UVA) e especializada em Planejamento Educacional pela Universidade Salgado de Oliveira (UNIVERSO), em Gestão Escolar Progestão (UDESC e UECE), em Gestão e Avaliação da Educação Pública (UFJF/CAEd) e em Formação de Professores para o Ensino Superior(FJN). É mestre pelo em Gestão e Avaliação da Educação Pública pelo Programa de Pós-Graduação Profissional da Universidade Federal de Juiz de Fora (UFJF/CAEd).Atualmente trabalha na Secretaria de Educação do Estado do Ceará . Faculdade de Juazeiro do NorteFJN. Departamento de Enfermagem. professoraednabelem@gmail.com
} 


\section{Introdução}

As transformações ocorridas no âmbito de atuação do profissional farmacêutico provocaram mudanças em sua formação acadêmica, tendo em vista a necessidade de aproximá-lo das necessidades da população, utilizando-se do medicamento como um meio e o paciente como o centro do cuidado, bem como pelo fato da sua atuação de forma integrada à equipe de saúde, necessitando, portanto, de habilidades como liderança, proatividade e trabalho em grupo.

No intuito de promover o desenvolvimento dessas habilidades, o Artigo $2^{\circ}$ das Diretrizes Curriculares Nacionais para o curso de graduação em Farmácia diz que as estratégias utilizadas para a formação devem ser centradas na aprendizagem do estudante, tendo o professor como mediador e facilitador desse processo (BRASIL, 2017).

Nesse processo de mudança de paradigma entre a formação baseada na escola tradicional e o uso de metodologia de aprendizagem ativa destaca-se a "Team-Based Learning" (TBL) ou Aprendizagem Baseada em Equipes (ABE).

A TBL utiliza uma estratégia instrucional que estimula o aluno a desenvolver, processar e maximizar a discussão intelectual e a dinâmica de equipe, ou seja, sua fundamentação teórica é baseada no construtivismo e na resolução de problemas (FATMI et al., 2013).

A referida estratégia de ensino foi desenvolvida, no início de 1990, pelo Professor Larry Michaelsen no curso de Administração de Empresas da Universidade de Oklahoma (EUA) em resposta a quatro fatos principais: 1) Aumento do tamanho da sua classe; 2) próprio descontentamento com as suas palestras; 3) a incapacidade de saber o que e como pensavam seus alunos durante as aulas; e 4) o fato de que os alunos não têm oportunidades para resolverem, em sala de aula, outros problemas que seriam resolvidos no mundo real dos negócios. Tudo isso o fez pensar que a manutenção de um ensino tradicional centrado no professor e nos conteúdos seria um desperdício de tempo (PARMELEE et al., 2012).

Segundo o professor Michaelsen et al., (2002), autor da estratégia ativa de ensino, os quatro princípios fundamentais da TBL são: 
I) Equipes: As equipes têm número limitado de alunos, devendo ser formadas pelo professor, e precisam ser permanentes para aperfeiçoarem as funções de coesão e de desenvolvimento de aprendizagem.

II) Fase 1: As equipes são preparadas durante o processo de aprendizagem inicial como garantia para alcançarem o sucesso subsequente de atividades de execução.

III) Fase 2: As equipes passam a maior parte do tempo de aula testando os seus conhecimentos em atividades de execução. As equipes estão aprendendo e condicionadas para resolverem problemas autênticos, interessantes e do mundo real, aplicando na prática os conceitos e ideias. Assim, os alunos tomam decisões complexas, fazem relatórios públicos e recebem feedback de qualidade e imediato.

IV) Responsabilidade: Os alunos são encorajados a serem responsáveis pelo seu preparo através de leituras anteriores e individuais, no início de testes de classe, e continuam a contribuir para o sucesso da equipe durante o teste. A revisão ou avaliação pelos pares revela-se um elemento essencial para motivar a responsabilidade e a participação dos alunos em equipes.

As sessões de TBL começam com um teste individual de avaliação rápida, previamente elaborada, em seguida, o mesmo é respondido em equipe. O objetivo das discussões em grupo é a troca de experiências, de modo que todos devem chegar a um consenso sobre as questões da avaliação. Após chegarem a um consenso, as equipes se reúnem como uma classe e revelam, ao mesmo tempo, suas respostas. Estas, então, se tornam o material para a discussão em classe, durante a qual os principais pontos de ensino são revistos (RAVINDRANATH et al., 2010).

Diante do contexto do uso de metodologias ativas na formação dos profissionais de saúde, objetivou-se avaliar na presente pesquisa o aproveitamento de discentes mediante a utilização da estratégia de "Team-Based Learning" (TBL) em disciplina do primeiro e do último ano da graduação em Farmácia em uma Instituição de Ensino Superior da região do Cariri cearense. 


\section{Métodos}

Trata-se de um estudo do tipo retrospectivo, baseado na análise documental dos registros das fichas de avaliação através da estratégia de TBL aplicada em disciplinas ministradas no primeiro ano e no último ano de graduação em Farmácia. As disciplinas selecionadas foram aquelas ministradas pela pesquisadora.

O levantamento dos dados ocorreu em uma Instituição de Ensino Superior (IES) do interior do estado do Ceará durante o mês de janeiro de 2017, referente ao semestre de 2016.2, período em que foi aplicada a estratégia de aprendizagem em uma disciplina do segundo semestre e do nono semestre, ou seja, primeiro e último ano de graduação em Farmácia, respectivamente.

A divisão dos discentes em grupo foi realizada pelo docente-pesquisador no intuito de garantir a heterogeneidade dos componentes, sendo cada equipe composta por 05 alunos. Totalizaram-se 04 equipes no primeiro e último ano de graduação em Farmácia. O conteúdo abordado na avaliação, utilizando-se da estratégia TBL, foi indicado pelo docente-pesquisador com uma semana de antecedência da realização da atividade.

Os dados obtidos foram tratados através de estatística simples, utilizando-se do software Excel versão 2013 que possibilitou o cálculo das médias aritméticas e a construção de gráficos e tabelas.

\section{Resultados e Discussão}

Através da coleta de dados, traçou-se um paralelo comparativo do aproveitamento do conteúdo abordado na TBL de maneira individual e em equipe, bem como entre os semestres, distintamente. Para tanto, realizou-se o cálculo de média aritmética dos quesitos corretos registrados nos gabaritos individuais e em grupo. A tabela 1 apresenta o rendimento dos discentes do primeiro ano de graduação frente ao assunto abordado pela estratégia em sala de aula. 
Tabela 1. Rendimento Individual e em equipe dos discentes de Farmácia do primeiro ano de graduação.

\begin{tabular}{|c|c|c|c|c|c|}
\hline EQUIPE & ALUNO & $\begin{array}{l}\text { AVALIAÇÃO } \\
\text { INDIVIDUAL }\end{array}$ & $\begin{array}{l}\text { AVALIAÇÃO } \\
\text { EM EQUIPE }\end{array}$ & $\begin{array}{c}\text { MÉDIA } \\
\text { ARITIMÉTICA }\end{array}$ & RENDIMENTO \\
\hline A & $\begin{array}{l}1 \\
2 \\
3 \\
4 \\
5 \\
\end{array}$ & $\begin{array}{l}6,5 \\
5,0 \\
5,0 \\
5,5 \\
5,5 \\
\end{array}$ & 7,5 & $\begin{array}{l}7,00 \\
6,25 \\
6,25 \\
6,50 \\
6,50 \\
\end{array}$ & $\begin{array}{l}+0,50 \\
+1,25 \\
+1,25 \\
+1,00 \\
+1,00\end{array}$ \\
\hline B & $\begin{array}{c}6 \\
7 \\
8 \\
9 \\
10\end{array}$ & $\begin{array}{l}7,0 \\
6,5 \\
6,0 \\
9,5 \\
4,5\end{array}$ & 8,0 & $\begin{array}{l}7,50 \\
7,25 \\
7,00 \\
8,75 \\
6,25\end{array}$ & $\begin{array}{l}+0,50 \\
+0,75 \\
+1,00 \\
-0,75 \\
+1,75\end{array}$ \\
\hline $\mathrm{C}$ & $\begin{array}{l}11 \\
12 \\
13 \\
14 \\
15 \\
\end{array}$ & $\begin{array}{l}6,0 \\
6,5 \\
4,5 \\
5,5 \\
4,0 \\
\end{array}$ & 8,5 & $\begin{array}{l}7,25 \\
7,50 \\
6,50 \\
7,00 \\
6,25 \\
\end{array}$ & $\begin{array}{l}+1,25 \\
+1,00 \\
+2,00 \\
+1,50 \\
+2,25\end{array}$ \\
\hline D & $\begin{array}{l}16 \\
17 \\
18 \\
19 \\
20\end{array}$ & $\begin{array}{l}5,5 \\
8,0 \\
6,5 \\
5,0 \\
8,0\end{array}$ & 9,0 & $\begin{array}{l}7,25 \\
8,50 \\
7,75 \\
7,00 \\
8,50\end{array}$ & $\begin{array}{l}+1,75 \\
+0,50 \\
+1,25 \\
+2,00 \\
+0,50\end{array}$ \\
\hline
\end{tabular}

Fonte: Própria Pesquisa.

$\mathrm{Na}$ disciplina do primeiro ano de graduação em Farmácia verificou-se um acréscimo de 1,11 pontos do aproveitamento da avaliação individual do aluno para a avaliação em equipe, variando entre discentes que tiveram resultado negativo e aqueles que aumentaram em 2,00 pontos o seu aproveitamento. $\mathrm{O}$ caso do aluno número 9 destaca-se pelo seu elevado rendimento individual e decréscimo quando da avaliação em equipes. Presume-se que este discente tenha apresentado dificuldades em discutir em equipe seu ponto de vista frente às questões discutidas. Situações como estas variam desde a timidez até a insegurança do seu próprio conhecimento.

Para o Conselho Nacional de Educação (BRASIL, 2001), a aprendizagem deve ser baseada no diálogo e na interação entre os alunos, o que contempla as habilidades de comunicação e trabalho colaborativo em equipes que serão necessárias ao futuro profissional e respondem às Diretrizes Curriculares Nacionais brasileiras. 
Tabela 2. Rendimento individual e em equipe dos discentes de Farmácia do último ano de graduação.

\begin{tabular}{|c|c|c|c|c|c|}
\hline EQUIPE & ALUNO & $\begin{array}{l}\text { AVALIAÇÃO } \\
\text { INDIVIDUAL }\end{array}$ & $\begin{array}{l}\text { AVALIAÇÃO } \\
\text { EM EQUIPE }\end{array}$ & $\begin{array}{c}\text { MÉDIA } \\
\text { ARITIMÉTICA }\end{array}$ & RENDIMENTO \\
\hline A & $\begin{array}{l}1 \\
2 \\
3 \\
4 \\
5 \\
\end{array}$ & $\begin{array}{l}6,0 \\
3,0 \\
4,5 \\
4,5 \\
2,5 \\
\end{array}$ & 6,0 & $\begin{array}{l}6,00 \\
4,50 \\
5,25 \\
5,25 \\
4,25\end{array}$ & $\begin{array}{l}+0,00 \\
+1,50 \\
+0,75 \\
+0,75 \\
+1,75\end{array}$ \\
\hline B & $\begin{array}{c}6 \\
7 \\
8 \\
9 \\
10\end{array}$ & $\begin{array}{l}4,0 \\
3,0 \\
6,5 \\
1,0 \\
4,5\end{array}$ & 4,0 & $\begin{array}{l}4,00 \\
3,50 \\
5,25 \\
2,50 \\
4,25\end{array}$ & $\begin{array}{l}+0,00 \\
+0,50 \\
-1,25 \\
+1,50 \\
-0,25\end{array}$ \\
\hline $\mathrm{C}$ & $\begin{array}{l}11 \\
12 \\
13 \\
14 \\
15 \\
\end{array}$ & $\begin{array}{l}8,0 \\
5,0 \\
3,0 \\
3,0 \\
4,5 \\
\end{array}$ & 8,5 & $\begin{array}{l}8,25 \\
6,75 \\
5,75 \\
5,75 \\
6,50 \\
\end{array}$ & $\begin{array}{l}+0,25 \\
+1,75 \\
+2,75 \\
+2,75 \\
+2,00\end{array}$ \\
\hline $\mathrm{D}$ & $\begin{array}{l}16 \\
17 \\
18 \\
19 \\
20\end{array}$ & $\begin{array}{l}4,0 \\
5,0 \\
5,5 \\
5,5 \\
3,5\end{array}$ & 7,0 & $\begin{array}{l}5,50 \\
6,00 \\
6,25 \\
6,25 \\
5,25\end{array}$ & $\begin{array}{l}+1,50 \\
+1,00 \\
+0,75 \\
+0,75 \\
+1,75\end{array}$ \\
\hline
\end{tabular}

Fonte: Própria Pesquisa.

Na disciplina do último ano de graduação em Farmácia verificou-se um acréscimo de 1,02 pontos do aproveitamento da avaliação individual do aluno para a avaliação em equipe, conforme a figura 1. Destaca-se a equipe B com rendimento bastante insatisfatório, bem como a média geral do aproveitamento individual dos alunos que foi de apenas 3,92 pontos corretos. Nessa situação é possível que os discentes não tenham cumprido a etapa de preparação para realização da TBL, ou seja, o estudo prévio do assunto abordado pela estratégia de aprendizagem, conforme orientada pelo docente. 


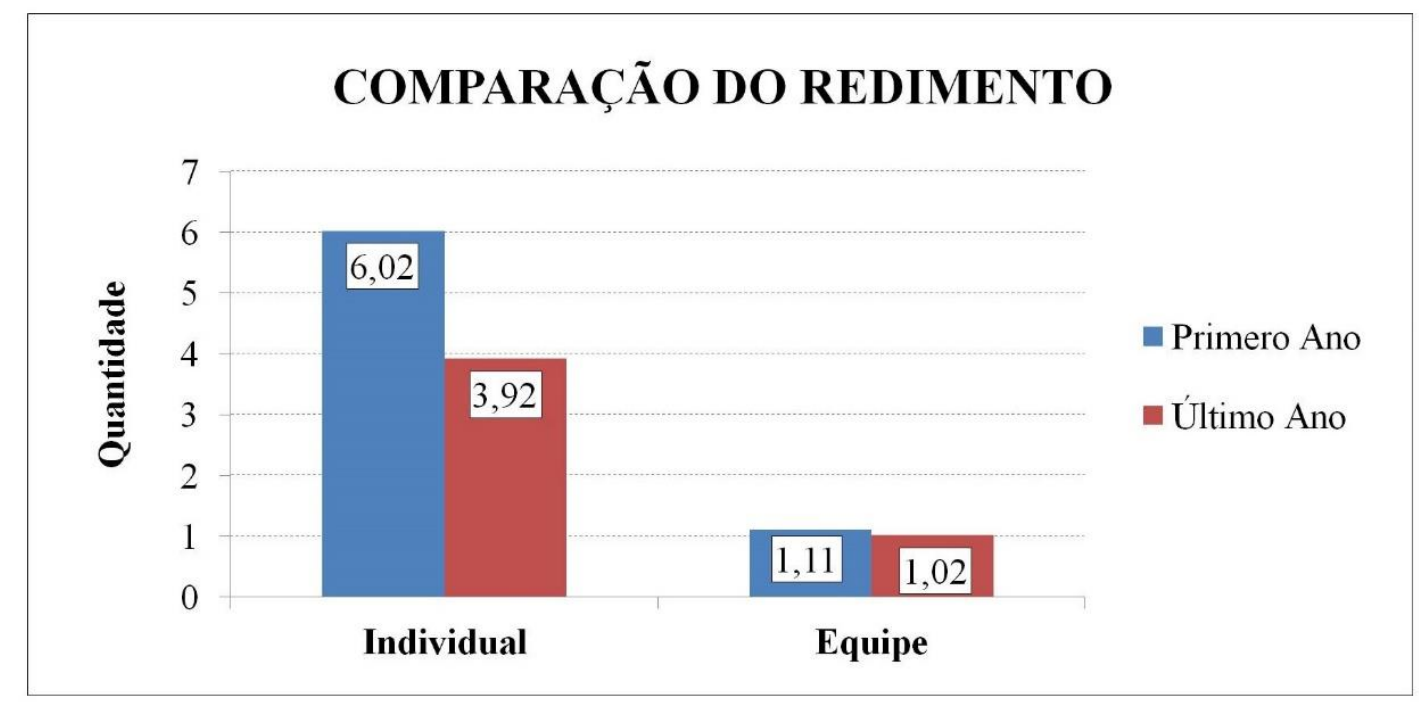

Figura 1. Comparação do rendimento, mediante aplicação da TBL, entre discentes do primeiro ano e do último ano de graduação em Farmácia.

Fonte: Própria Pesquisa.

Para Michaelsen et al., (2002), a preparação da atividade individual pré-classe é uma etapa crítica. Se os alunos individualmente não completam as tarefas pré-classe, eles não serão capazes de contribuir para o desempenho de sua equipe. A falta desta preparação dificulta o desenvolvimento de coesão do grupo e resulta em ressentimento dos alunos que se prepararam, pois estes percebem a sobrecarga causada pelos seus colegas menos dispostos e/ou menos capazes.

\section{Conclusão}

Sugere-se que o docente ao optar pela estratégia de TBL compreenda seus princípios, bem como realize um processo de sensibilização eficiente dos discentes, pois acredita-se que o decréscimo do rendimento em equipe dos alunos do último ano de graduação em Farmácia tenha ocorrido por falhas nesse processo.

O docente deve ser capaz de inovar as abordagens em sala de aula para que o discente se sinta constantemente motivado e disposto a encarar novos desafios, como também preparar-se para trabalhar em equipe e contribuir efetivamente para o sucesso da mesma. 
Dessa forma, este aluno terá maiores possibilidades de se tornar um profissional alinhado com as necessidades atuais do mundo do trabalho.

\section{Referências}

BRASIL. Ministério da Educação. Resolução CNE/CES N 4, de 7 de Novembro de 2001. Institui Diretrizes Curriculares Nacionais do Curso de Graduação em Medicina. Brasília: Distrito Federal. Diário Oficial da União, 2001. Disponível em: http://portal.mec.gov.br/cne/arquivos/pdf/CES04.pdf.

BRASIL. Ministério da Educação. Resolução CES Nº 202, de 20 de Outubro de 2017. Institui as Diretrizes Curriculares Nacionais do Curso de Graduação em Farmácia e dá outras providências. Brasília: Distrito Federal. Diário Oficial da União, 2017. Disponível em: http://www.semesp.org.br/site/wp-content/uploads/2017/10/COMUNICADO-JURIDICORESOLU\%C3\%87\%C3\%83O-CES-N\%C2\%BA-6-DE-19-DE-OUTUBRO-DE-2017.pdf.

FATMI, M.; HARTLING, L.; HILlIER, T.; CAMPBELL, S.; OSWALD, A. E. The Effectiveness of Team-Based Learning on Learning Outcomes in Health Professions Education: BEME Guide No. 30. Med Teach, v. 35, n. 12, p. e1608-1624, 2013. DOI: 10.3109/0142159X.2013.849802.

MICHAELSEN, L. K.; KNIGHT, A. B.; FINK, L. D. Team-Based Learning: A Transformative Use of Small Groups. $1^{\text {a }}$ Edição, Praeger, 2002.

PARMELEE, D.; MICHAELSEN, L. K.; COOK, S.; HUDES, P, D. Team-Based Learning: A Practical Guide: AMEE Guide No. 65. Med Teach, v. 34, n. 5, p. e275-e287, 2012. DOI: 10.3109/0142159X.2012.651179.

RAVINDRANATH, D.; GAY, T. L.; RIBA, M. B. Trainees as Teachers in Team-Based Learning. Acad Psychiatry, v. 34, n.4, p.294-297, 2010. DOI: 10.1176/appi.ap.34.4.294.

\section{Como citar este artigo (Formato ABNT):}

SARAIVA, Emanuela M.S.; ALENCAR, Gustavo de O.; MACHADO, Yanaina S. O. ; GOMES, Antônia E.B. Uso da Estratégia Aprendizagem Baseada em Equipes em Disciplinas da Graduação em Farmácia. Id on Line Revista ultidisciplinar e de Psicologia, 2018, vol.12, n.39, p.831-838. ISSN: 1981-1179.

Recebido: 06.11.2017

Aceito: 30.01 .2018 